\title{
Efficiency measurement strategy for a planetary gearbox with 2 degrees of freedom
}

\author{
Florian Verbelen ${ }^{a, b}$, Pieter Defreyne $e^{a, b}$, Peter Sergeant ${ }^{a, b}$ and Kurt Stockman ${ }^{a, b}$ \\ ${ }^{a}$ Department of Electrical Energy, Metals, Mechanical Constructions and Systems, Ghent University, \\ Belgium \\ ${ }^{b}$ Member of Energy Efficient Drive Trains partner of Flanders Make, www.eedt.ugent.be
}

\begin{abstract}
This paper discusses a method to calculate the efficiency of a planetary gearbox with 2 degrees of freedom. Standard measurement procedures in which the efficiency is measured as function of the load torque and input speed become infeasible due to the many possible input output configurations. To solve this, a method is proposed that combines the theory of virtual power and a limited set of measurements. The theory of virtual power is used to link the considered power flow in the device with an expression to calculate the efficiency of the planetary gear in terms of the efficiency of the power path between sun \& carrier and carrier \& ring. The efficiency of the aforementioned power paths is shown to depend only on the torque applied to these paths which means that speed dependent losses such as churning losses are negligible. Measurements of the efficiency as function of the torque applied to the sun for a varying speed ratio between ring and carrier are added to validate the approach.
\end{abstract}

\section{Introduction}

Due to increasing energy prices, the efficiency of drive trains and their individual components has gained a lot of interest. By optimizing the drive train as a whole, large savings on the long term are possible. This is called the Extend Product Approach (EPA). However, to apply EPA, knowledge of the energy efficiency of all drive train components over a wide range of operating points is vital.

For electrical components, measurement procedures and models are available to provide this important data as function of the operating point. In contrast to the latter, the provided efficiency of mechanical components is often restricted to a single operating point. In the last 5 years, a lot of effort has been spent in setting up measurement guidelines for mechanical gearboxes. The efficiency is measured and afterwards visualized as function of input speed and output torque [1], [2]. An important remark is that this method was developed for single input single output devices in which a unique power path can be defined. If another shaft is added, the method still holds, but the number of measurements to obtain an accurate description of the efficiency becomes unacceptably high. Moreover, visualizing efficiency or power losses on a 2D map is no longer possible. An example of such a gearbox is a 2 Degrees Of Freedom (DOF) planetary gearbox which is for instance used in hybrid electrical vehicles [3], wind turbines [4], aerospace [5], etc. Their main advantages are high efficiency, compactness and axisymmetric arrangement.

A lot of effort has already been spent to model the efficiency of the planetary gearbox. Pennestri has summarized all the work concerning efficiency evaluation in an interesting review [6]. The paper shows that the efficiency of a planetary gearbox, given a specific operating point, is determined based on the power flow (related to the operating point) and a fix efficiency value for the power path between sun \& carrier and carrier \& ring, denoted by path 1 and path 2 in Fig. 1, respectively. Moreover, it is shown that, although there are a lot of different mathematical expressions to calculate the efficiency, the different approaches are numerically equivalent. A completely novel approach to calculate the efficiency, based on virtual power, is introduced by Chen [7]-[9]. This approach has also been validated in [10] but rather on a limited dataset.

In this paper, the work of Chen [8] is used to derive equations to estimate the overall efficiency based on the power flows and efficiency values for the different/individual power paths. The individual efficiency values for the considered power paths are determined for 3 levels of accuracy: as function of load torque and input speed, purely depending on the load torque and finally they are assumed to be constant (identical to the approach in [8]). The calculated planetary gear efficiency, based on the different formulations of the efficiency of the power paths, is then compared with the measurements to determine what level of accuracy is required. 
This paper is structured as follows. In section 2, the equations to calculate the efficiency of the planetary gear are considered after which in section 3 the measurement setup is introduced. The results of the efficiency maps of power path 1 and 2 are shared in section 4 while in section 5 the impact of the different efficiency formulations of the power paths on the total gear train efficiency is discussed. Finally in section 6 the conclusions of the research are formulated.

\section{Theoretical model}

The considered planetary gear consists of a sun, carrier with planets and a ring, organized as in Fig. 1. The main parameters of the planetary gear can be found in Table 1.

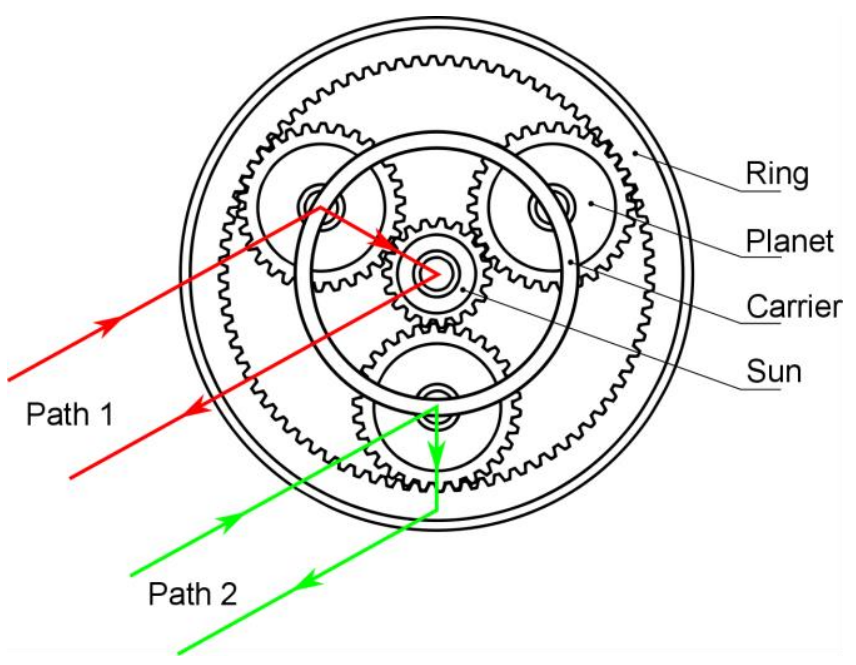

Figure 1: Principle scheme of the planetary gear.

Table 1: Number of teeth of the elements.

\begin{tabular}{|l|l|}
\hline Number of teeth of the sun $N s$ & 54 \\
\hline Number of teeth of the planets $N_{p}$ & 26 \\
\hline Number of teeth of the ring $N_{r}$ & 108 \\
\hline Maximum torque at the sun $T_{s, \max }$ & $45 \mathrm{Nm}$ \\
\hline Maximum torque at the carrier $T_{c, \max }$ & $137 \mathrm{Nm}$ \\
\hline Maximum torque at the $\operatorname{ring} T_{r, \max }$ & $90 \mathrm{Nm}$ \\
\hline
\end{tabular}

As described in [8], it is possible to derive the efficiency of the 2-DOF planetary gearbox as function of the power flow $\left(P_{s}, P_{c}, P_{r}\right)$, speed ratio between carrier and sun $\left(\frac{\omega_{c}}{\omega_{s}}\right)$ and 2 efficiency values that describe the efficiency of power path 1 and $2\left(\eta_{1}, \eta_{2}\right)$. Deriving the equations for the considered planetary gear is straightforward based on the content of [8] as all the equations of a similar planetary gear are derived in that paper. Hence, only the definition of parameter $C_{2}$ (see (6)) is different compared to the original paper which is due to the slightly different configuration of the planetary gear.

Table 2, gives an overview of all the possible equations to calculate the efficiency. The first step in selecting the correct equation is to determine the power flow. Based on the power flow, it is possible to 
select a row in Table 2. If, for example, the power flows from the sun $\left(P_{s}<0\right)$ to the carrier $\left(P_{c}>0\right)$ and ring $\left(P_{r}>0\right)$, row 3 is selected. The second step is to select the correct subcase (A or B). The conditions for these subcases are given in Table 3 and depend on the speed ratio between carrier and sun. For more information concerning the derivation of the subcases and separate efficiency equations, the reader is referred to [8].

Table 2: Equations to calculate the efficiency as function of the power flow and a given subcase.

\begin{tabular}{|c|c|c|}
\hline & Subcase A & Subcase B \\
\hline$P_{s}>0, P_{c}>0$ and $P_{r}<0$ & $\eta=\frac{1-A}{1-A+A C_{2}}$ & $\eta=\frac{1}{1-A C_{2}}$ \\
\hline$P_{s}>0, P_{c}<0$ and $P_{r}>0$ & $\eta=\frac{-C_{1}+A C_{1}+C_{2}-A C_{1} C_{2}}{-C_{1}+A C_{1}+C_{2}}$ & $\eta=\frac{C_{1}-C_{2}+A C_{2}-A C_{1} C_{2}}{C_{1}-C_{2}+A C_{2}}$ \\
\hline$P_{s}<0, P_{c}>0$ and $P_{r}>0$ & $\eta=\frac{1}{1-A C_{1}}$ & $\eta=\frac{1-A}{1-A+A C_{1}}$ \\
\hline$P_{s}>0, P_{c}<0$ and $P_{r}<0$ & $\eta=1-A C_{1}$ & $\eta=\frac{-1+A-A C_{1}}{-1+A}$ \\
\hline$P_{s}<0, P_{c}>0$ and $P_{r}<0$ & $\eta=\frac{C_{1}-A C_{1}-C_{2}}{C_{1}-C_{2}-A C_{1}+A C_{1} C_{2}}$ & $\eta=\frac{-C_{1}-A C_{1}+C_{2}}{-C_{1}+C_{2}-A C_{2}+A C_{1} C_{2}}$ \\
\hline$P_{s}<0, P_{c}<0$ and $P_{r}>0$ & $\eta=\frac{-1+A-A C_{2}}{-1+A}$ & $\eta=1-A C_{2}$ \\
\hline
\end{tabular}

In which:

$$
\begin{gathered}
A=\lambda_{1}+\lambda_{2}-\lambda_{1} \lambda_{2} \\
\lambda_{1}=1-\eta_{1}\left(T_{s}, \omega_{p}\right) \\
\lambda_{2}=1-\eta_{2}\left(T_{r}, \omega_{p}\right) \\
k=\frac{\omega_{c}}{\omega_{s}} \\
C_{1}=1-k \\
C_{2}=\frac{(k-1) N_{s}}{(k-1) N_{s}+k N_{r}}
\end{gathered}
$$

\begin{tabular}{|c|c|c|}
\hline & Subcase $A$ & Subcase B \\
\hline$P_{s}>0, P_{c}>0$ and $P_{r}<0$ & $k<0$ & else \\
\hline$P_{s}>0, P_{c}<0$ and $P_{r}>0$ & $\frac{N_{s}}{N_{r}+N_{s}}<k<1$ & else \\
\hline$P_{s}<0, P_{c}>0$ and $P_{r}>0$ & else & $0<k<\frac{N_{s}}{N_{r}+N_{s}}$ \\
\hline$P_{s}>0, P_{c}<0$ and $P_{r}<0$ & $0<k<\frac{N_{s}}{N_{r}+N_{s}}$ & else \\
\hline
\end{tabular}

Table 3: Conditions of the subcases. 


\begin{tabular}{|c|c|c|}
\hline$P_{s}<0, P_{c}>0$ and $P_{r}<0$ & $k>1$ & else \\
\hline$P_{s}<0, P_{c}<0$ and $P_{r}>0$ & else & $k<0$ \\
\hline
\end{tabular}

\section{Measurement setup}

The measurement setup consists of a planetary gear, a belt, 3 torque sensors and 3 electrical machines to load the shafts of the planetary gear. The purpose of the belt is to transmit torque from the ring to the load machine (and vise-versa). The torque sensors are used to measure the mechanical power going through each shaft. Each shaft can be fixed to go from a 2-DOF system to a 1-DOF system. These fixing mechanisms are used to block the sun and ring in 2 separate measurements in order to set up the 1-DOF efficiency maps of power path 1 and 2. Note that the efficiency map for the power path from carrier to ring includes the efficiency of the belt. The planetary gear system as such is thus the combination of the planetary gear component and the belt. During all measurements, the temperature has been controlled at $32 \pm 1^{\circ} \mathrm{C}$. This is of high importance due to the impact of temperature on the viscosity of the oil and thus the efficiency of the gearbox [11].

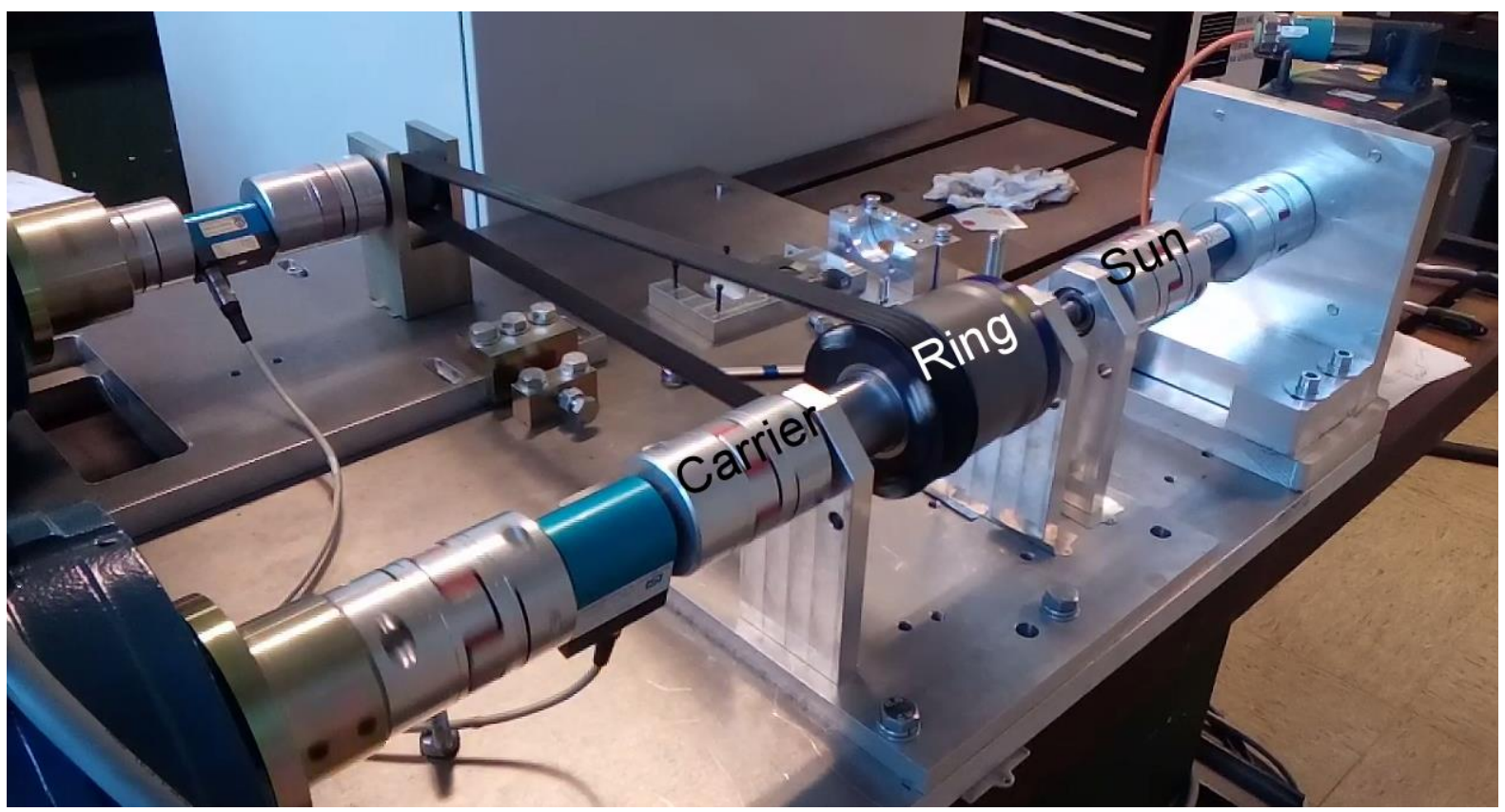

Figure 2: measurement setup

\section{a) Measurement accuracy}

The accuracy of the measured efficiency depends on the accuracy of the torque sensors and the $A D$ conversion of the signals. The used torque sensors are equipped with strain gauges and have a contactless signal transmission from rotor to stator. The details in terms of range and accuracy of the 3 torque sensors that are used can be found in Table 4 .

Table 4: Accuracy of the torque sensors.

\begin{tabular}{|l|l|l|l|}
\hline Range & Accuracy & Absolute fault & Location \\
\hline $50 \mathrm{Nm}$ & $0.2 \%$ full scale & $\pm 0.1 \mathrm{Nm}$ & Sun \\
\hline
\end{tabular}




\begin{tabular}{|l|l|l|l|}
\hline $100 \mathrm{Nm}$ & $0.1 \%$ full scale & $\pm 0.1 \mathrm{Nm}$ & Ring \\
\hline $200 \mathrm{Nm}$ & $0.1 \%$ full scale & $\pm 0.2 \mathrm{Nm}$ & Carrier \\
\end{tabular}

The accuracy of the $A D$ conversion is $0.11 \%$ full scale. The range goes from 0 to $5 \mathrm{~V}$ for the torque sensors with a range of 50 and $100 \mathrm{Nm}$. For the torque sensor with a range of $200 \mathrm{Nm}$, the output range is 0 to $10 \mathrm{~V}$.

To demonstrate how the Absolute Fault (AF) on an efficiency measurement can be calculated, an example is presented for when the ring is blocked. In this case, power flows from carrier to sun. The torque at the sun $T_{s}$ equals $26 \mathrm{Nm}$, at the carrier $T_{c}$ a torque of $80 \mathrm{Nm}$ is applied, the speed of the sun $\omega_{s}$ equals $792 \mathrm{rpm}$ and the speed of the carrier $\omega_{c}$ is equal to $264 \mathrm{rpm}$. The efficiency of this path can be calculated as:

$$
\eta=\frac{P_{\text {out }}}{P_{\text {in }}}=\frac{T_{s} \omega_{s}}{T_{c} \omega_{c}}=\frac{T_{s} \omega_{c} \frac{N_{s}+N_{r}}{N_{s}}}{T_{c} \omega_{c}}=\frac{T_{s}\left(N_{s}+N_{r}\right)}{T_{c} N_{s}}=97.5 \%
$$

The total uncertainty of this measurement depends on the uncertainty of the torque sensor and the uncertainty of the $A D$ conversion (output of the torque sensor is an analogue signal). The accuracy of the speed measurement has no impact on measured efficiency as can be seen in (8). The total Relative Fault (RF) and AF are calculated using the following equations:

$$
\begin{gathered}
R F(\text { tot })=\sqrt{R F\left(T_{s}\right)^{2}+R F\left(T_{c}\right)^{2}}=\sqrt{\left(\frac{A F\left(T_{s}\right)}{\left|T_{s}\right|}\right)^{2}+\left(\frac{A F\left(T_{c}\right)}{\left|T_{c}\right|}\right)^{2}} \\
A F(\text { tot })=R F(t o t) \eta
\end{gathered}
$$

According to Table 4, the AF on the torque sensor of the sun is $\pm 0.1 \mathrm{Nm}$ and of the carrier $\pm 0.2 \mathrm{Nm}$. The AF due to the $A D$ converter of the torque sensor of the sun is $5 \mathrm{~V} \times 11 \%= \pm 5.5 \mathrm{mV}$ which translates to $\pm 0.0275 \mathrm{Nm}$. The same can be done for the torque sensor mounted at the carrier which results in an AF of $\pm 0.22 \mathrm{mNm}$. Based on (9) it is now possible to calculate the total relative fault $R F($ tot $)$ :

$$
R F(t o t)=\sqrt{\left(\frac{0.1+0.055}{26}\right)^{2}+\left(\frac{0.2+0.22}{80}\right)^{2}}=0.79 \%
$$

which yields an $\mathrm{AF}$ of:

$$
A F(t o t)=\frac{0.79}{100} \times \frac{97.5}{100}=0.77 \%
$$

\section{Measurement of 1-DOF efficiency map}

In the following sections the results of the 1-DOF measurements of the 2 power paths are discussed. Note that only a limited dataset is presented in the paper. Hence, the variation of the efficiency with the speed of the planets is discussed based on 4 measurements. However, the real measured dataset covers the efficiency for 18 different speed values, each for 31 torque values.

\section{a) Path 1 (sun to carrier)}


As mentioned in (2), the efficiency $\eta_{1}$ is a function of torque and speed. The efficiency $\eta_{1}$ is related to the power path between the sun and carrier (see Fig. 1) and is written as function of the torque applied to the sun $T_{s}$ and the speed of the planets $\omega_{p}$. As, in normal operation, the power could split towards the ring, the ring is blocked which reduces the 2-DOF planetary gear to a 1-DOF system. Hence, the only power path which remains goes from the sun to the carrier (and vise-versa). Under the assumption that the direction of the power of the 1-DOF contacts has no impact on the efficiency, it does not matter which element (sun or planets) is chosen as input or output to measure the efficiency map. As the torque on the planets cannot be measured, the measurable quantity for the planets is speed. Therefore, the efficiency $\eta_{1}$ can be measured as function of the torque on the sun $T_{s}$ (output property) and the speed of the planets $\omega_{p}$ (input property).

Fig. 3 shows the efficiency as function of the torque applied to the sun for varying speed of the planets. As can be seen in the graph, the efficiency is fairly constant in a wide range of torque values. This can support the assumption of taking a constant value for $\eta_{1}$. However, at low torque, as expected, the efficiency drops. Furthermore, the dependency with the speed is very low and only noticeable at low torque.

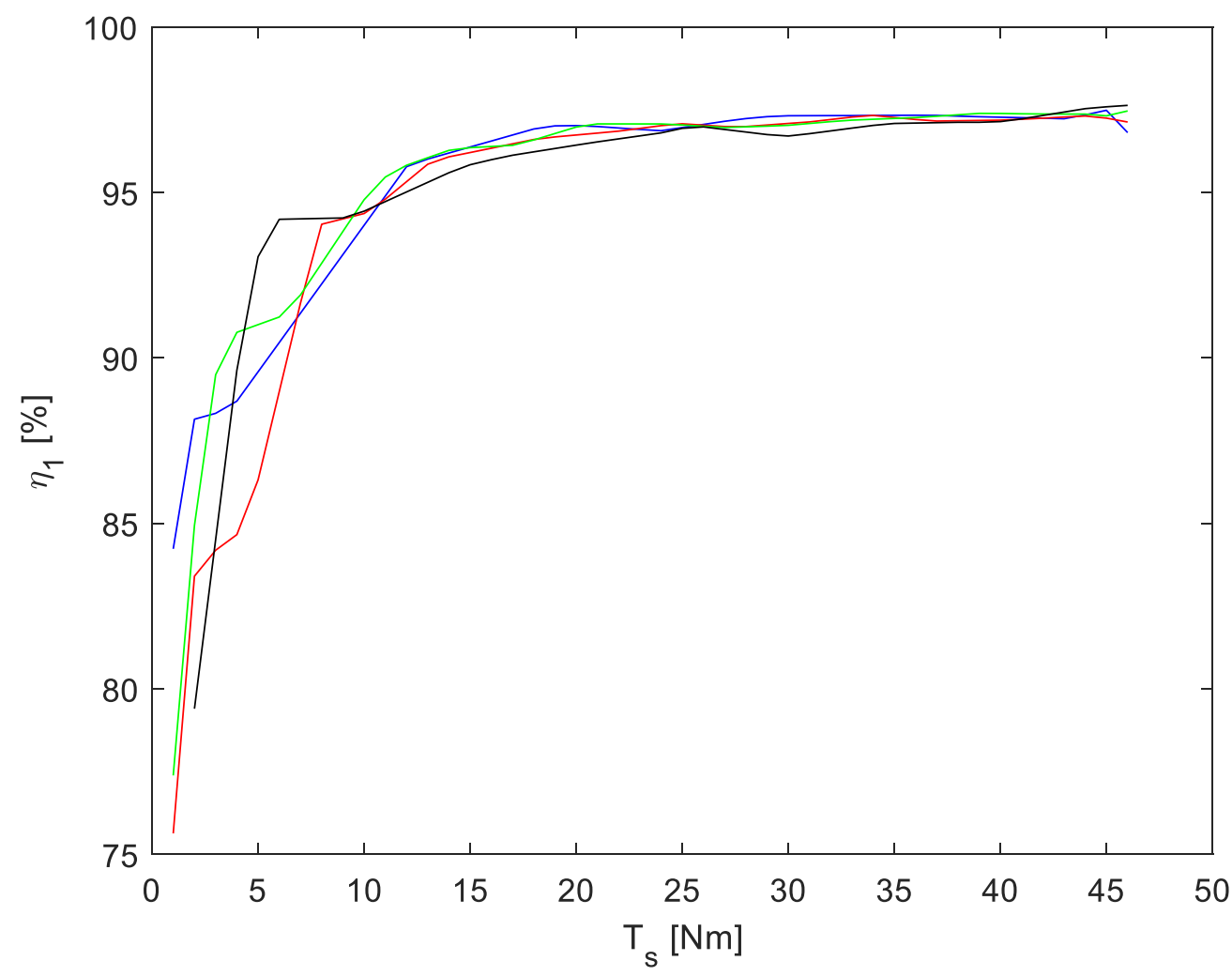

Figure 3: Efficiency of the path going from sun to carrier $\eta_{1}$ as function of the torque applied to the sun $T_{s}$ for varying speed of the planets $\omega_{p}$. Blue: $\omega_{p}=500 \mathrm{rpm}$, red: $\omega_{p}=$ 1000rpm, green: $\omega_{p}=1500 \mathrm{rpm}$, black: $\omega_{\mathrm{p}}=2000 \mathrm{rpm}$.

Based on the measured data (the complete dataset) it is possible to fit a surface (see Fig. 4). This theoretical expression is equal to:

$$
\eta_{1}=\left(a_{1}+b_{1} \omega_{p}\right) T_{s}^{\left(c_{1} \omega_{p}+d_{1}\right)}+e_{1}
$$


With $a_{1}=-7.558 \times 10^{-5}, b_{1}=-0.134, c_{1}=-5.326 \times 10^{-5}, d_{1}=-0.7706$ and $e_{1}=0.9836$. Note that this is an empirical formula which tends to be a good fit for most of the operating range. For extremely low torque values, (13) becomes useless as the efficiency goes to infinity. The advantage, however, is that, based on (13), it is easy to investigate the impact of reducing $\eta_{1}\left(T_{s}, \omega_{p}\right)$ to $\eta_{1}\left(T_{s}\right)$.

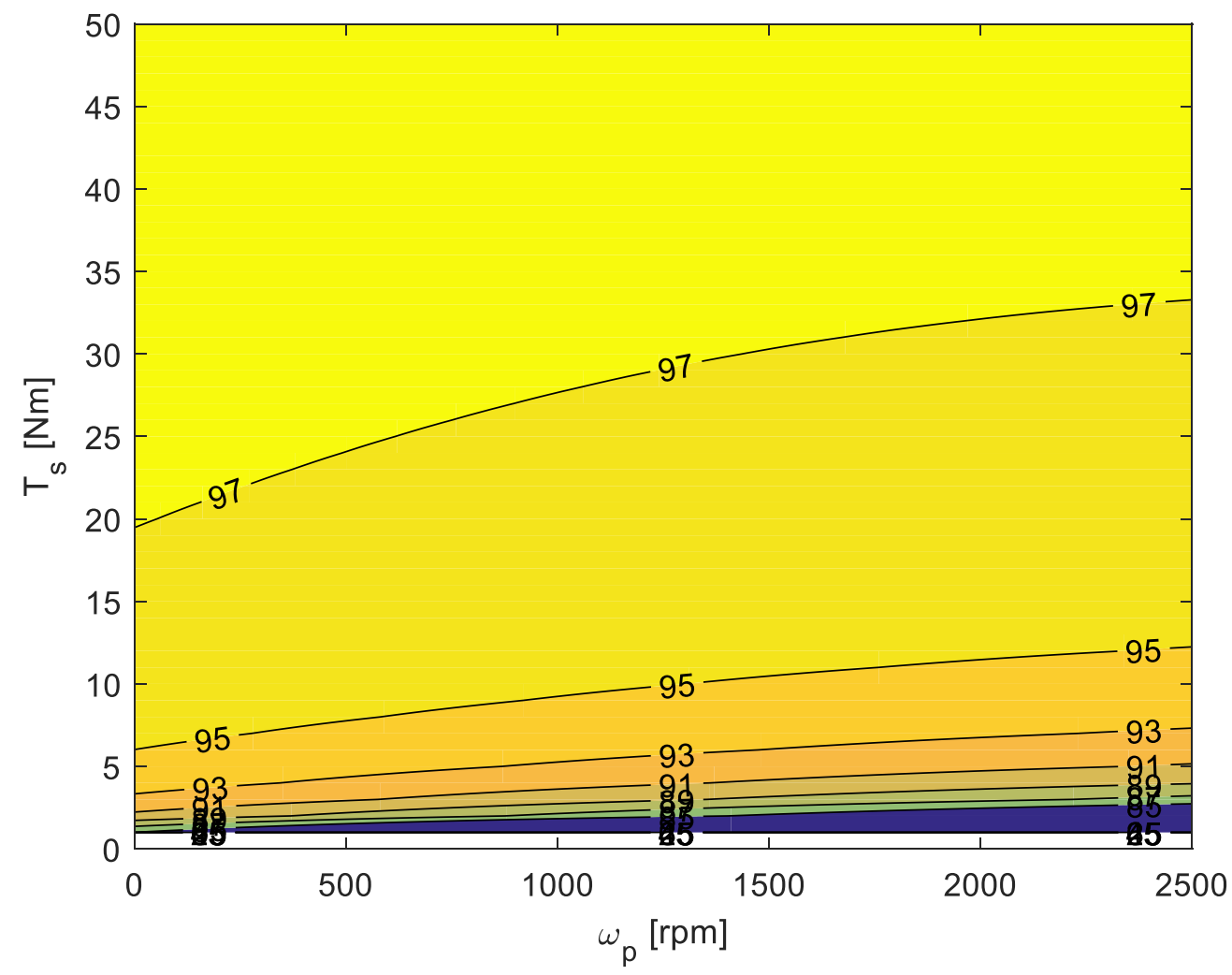

Figure 4: Fitted efficiency of power path 1 as function of the speed of the planets and torque applied to the sun

\section{b) Path 2 (carrier to sun)}

A similar methodology has been applied to measure the efficiency map of the second power path. Now the sun is fixed, allowing a unique power flow from carrier to ring. Fig. 5 shows the results as function of torque applied to the ring for varying speed of the planets. Again, the efficiency is rather constant and almost independent of the speed of the planets. Note that the efficiency is 1 to $2 \%$ lower compared to the power path from sun to carrier. One of the reasons for this lower efficiency is that the efficiency of power path 2 is the combination of the efficiency of the planetary gear and the belt. 


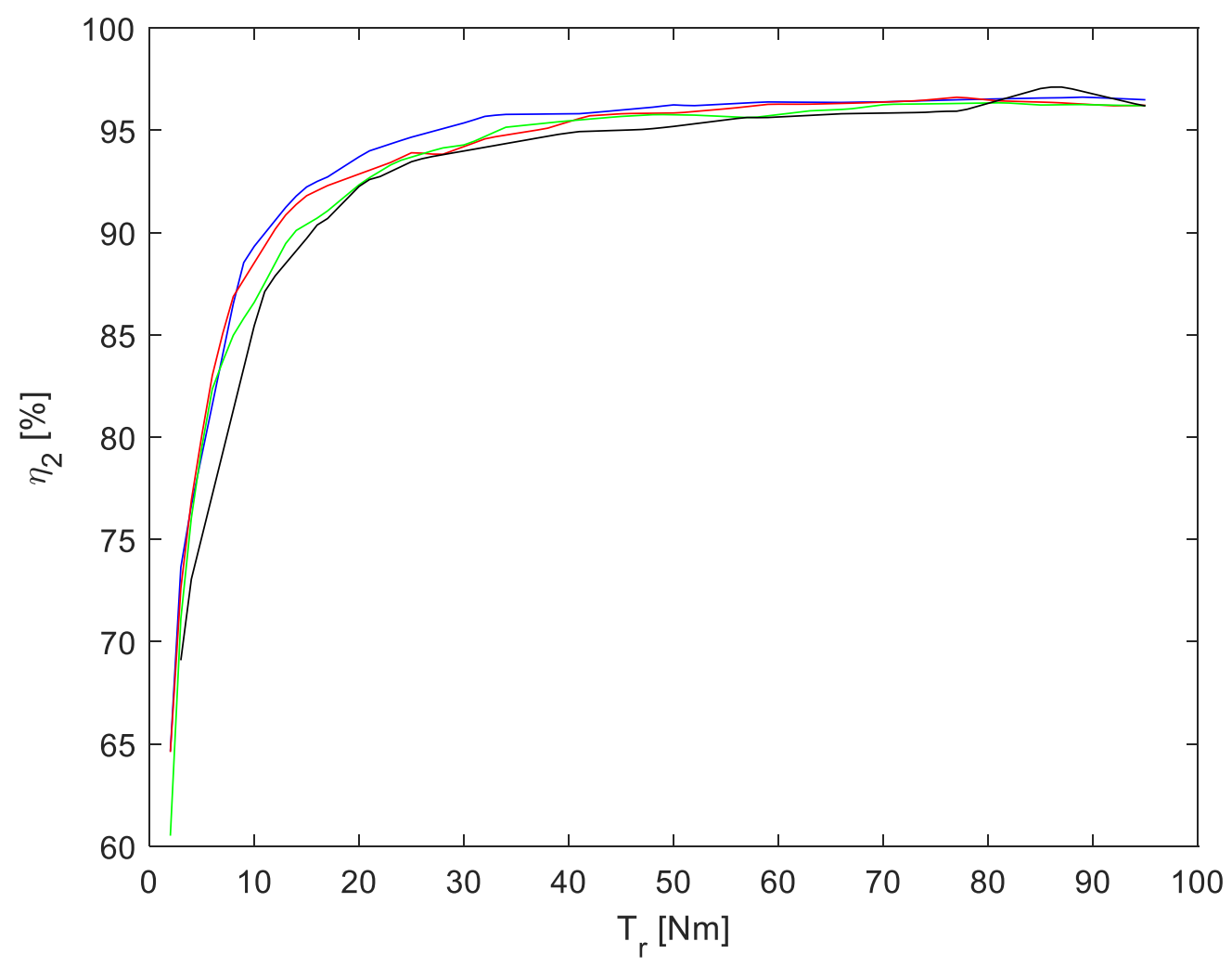

Figure 5: Efficiency of the path going from carrier to ring $\eta_{2}$ as function of the torque applied to the ring $T_{r}$ for varying speed of the planets $\omega_{p}$. Blue: $\omega_{p}=500 \mathrm{rpm}$, red: $\omega_{p}=$ 1000rpm, green: $\omega_{p}=1500 \mathrm{rpm}$, black: $\omega_{\mathrm{p}}=2000 \mathrm{rpm}$.

Based on the measured data it is again possible to fit a surface (see Fig. 6). This theoretical expression is equal to:

$$
\eta_{2}=\left(a_{2}+b_{2} \omega_{p}\right) T_{s}^{\left(c_{2} \omega_{p}+d_{2}\right)}+e_{2}
$$

With $a_{2}=-14.2 \times 10^{-5}, b_{2}=-0.5826, c_{2}=-2.337 \times 10^{-14}, d_{2}=-0.901$ and $e_{2}=0.9791$. 


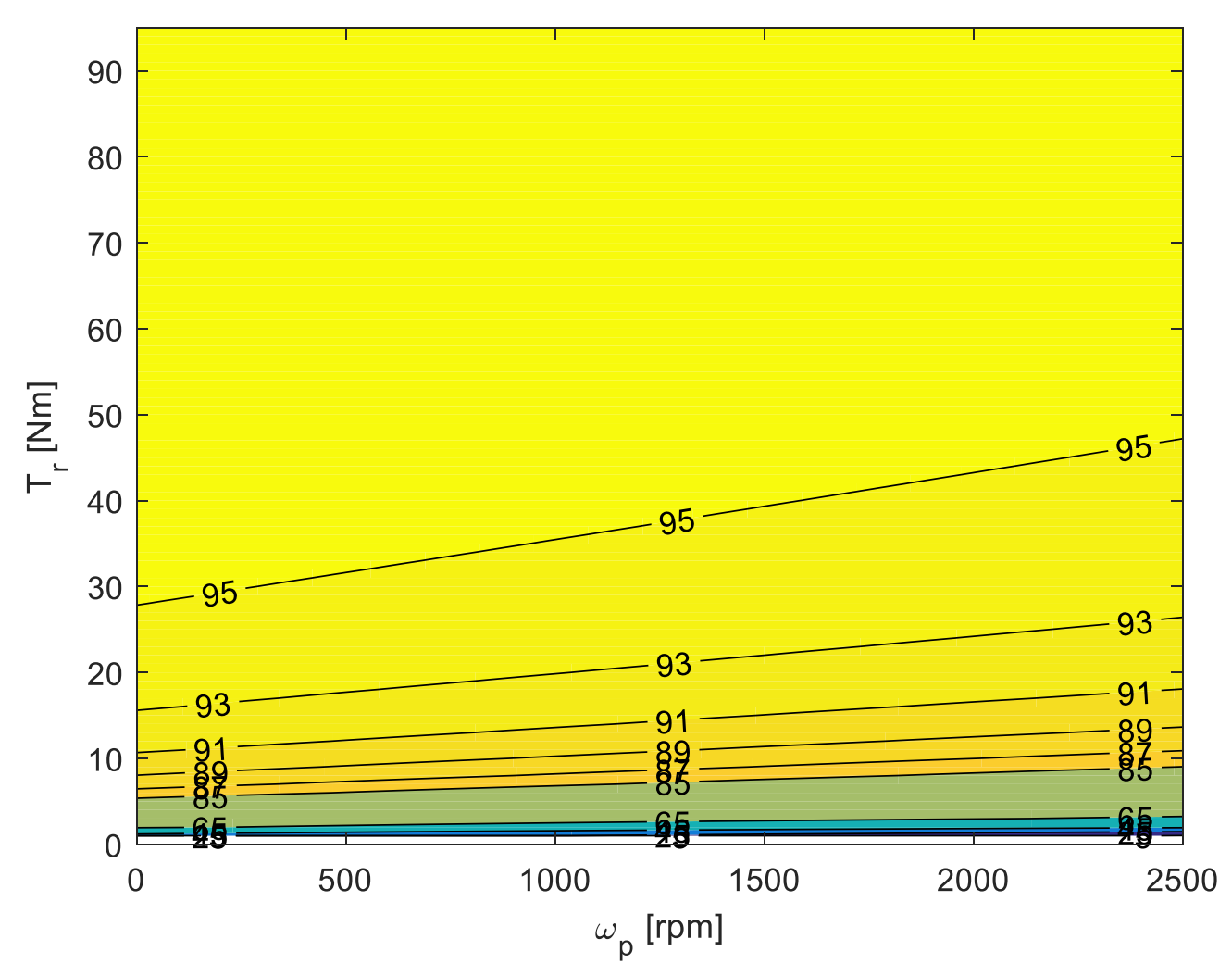

Figure 6: Fitted efficiency of power path 2 as function of the speed of the planets and torque applied to the ring.

\section{Case study}

Based on the equations written down in Table 1, (13) and (14), it is possible to calculate the efficiency for all possible operating points. Fig. 7 shows the measured efficiency values of the planetary gear as function of varying speed ratio (ratio of carrier speed and ring speed) and stator torque. The ring speed was chosen constant at 200rpm. Note that as all 3 shafts are rotating, speed cannot be excluded anymore from the equation to calculate the efficiency. Therefore, in contrast with the earlier explanation on the measurement accuracy, accuracy of the speed measurement does have an effect.

The results presented in Fig. 7 will be used as reference to benchmark the 3 cases: efficiency depending on both the load torque and input speed (case 1), efficiency as function of the load torque (case 2) and fixed efficiency (case 3). For case 1, (13) and (14) are used to calculate the efficiency of power path 1 and 2, denoted by $\eta_{1}$ and $\eta_{2}$, respectively. For case 2, (13) and (14) are again used but at a fixed speed of the planets. This speed is arbitrarily chosen at 500rpm. For case 3 , a fixed efficiency is chosen equal to $97 \%$ and $96 \%$ for $\eta_{1}$ and $\eta_{2}$, respectively. This decision is taken purely on the average efficiency in the operating range starting from $20 \mathrm{Nm}$ until the maximum torque. 


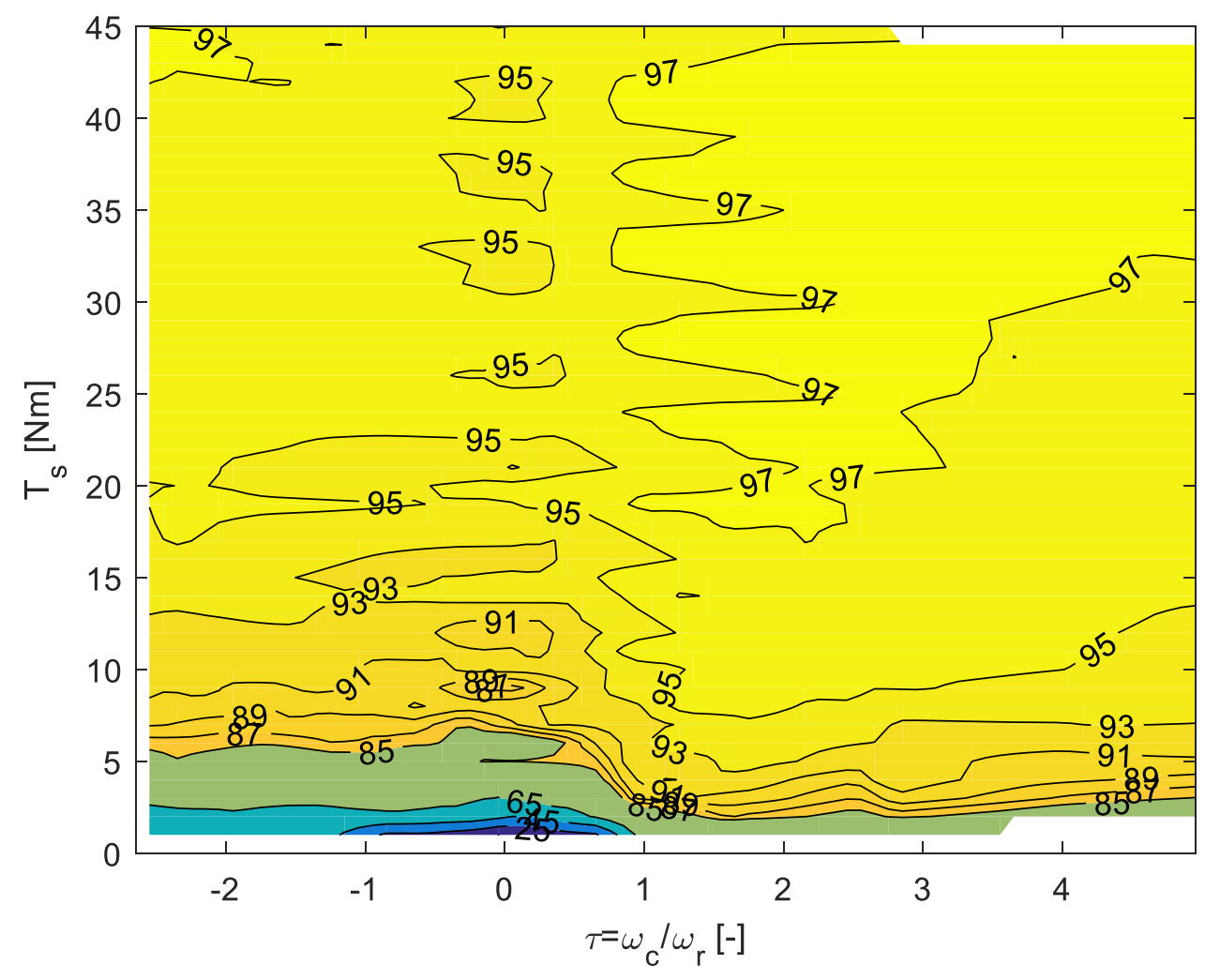

Figure 7: Measured efficiency of the planetary gearset as function of varying speed ratio $\tau=\frac{\omega_{c}}{\omega_{r}}$ and stator torque $T_{s}$.

Fig. 8 provides a comparison between the cases and the actual measured efficiency. The results show that the model is clearly capable of estimating the efficiency of the planetary gear, certainly for higher torque values.

Comparing the outcome based on case 1 and 2 shows that the results are almost identical. This means that there is no added value in measuring a complete efficiency map to resemble power path 1 and 2 as the variation of the efficiency with the speed is just too insignificant. However, there is a significant added value at low torque between case $2\left(\eta_{1,2}\right.$ vary as function of $\left.T_{s, r}\right)$ and case 3 ( $\eta_{1,2}=$ constant). At higher torque, $\eta_{1}$ and $\eta_{2}$ become fairly constant (see Fig. 4 and 6), which explains why the differences between case 2 and 3 diminish for higher torque.

Based on this analysis it is possible to conclude that the efficiency of a planetary gearbox can be estimate based on 2 measurements which take into account the efficiency of power path 1 and 2 as function of the load torque. For case 2, a complete efficiency map has been set up and is presented as Fig. 9. It's clear by comparing Fig. 7 and 9 that there is a close match between measurement and model results. The average mismatch (complete operating range) between estimation and measurement is $1.7 \%$. If torque values below $10 \mathrm{Nm}$ are excluded, the average difference is below $1 \%$ which is close to the error bands of the measurements. 

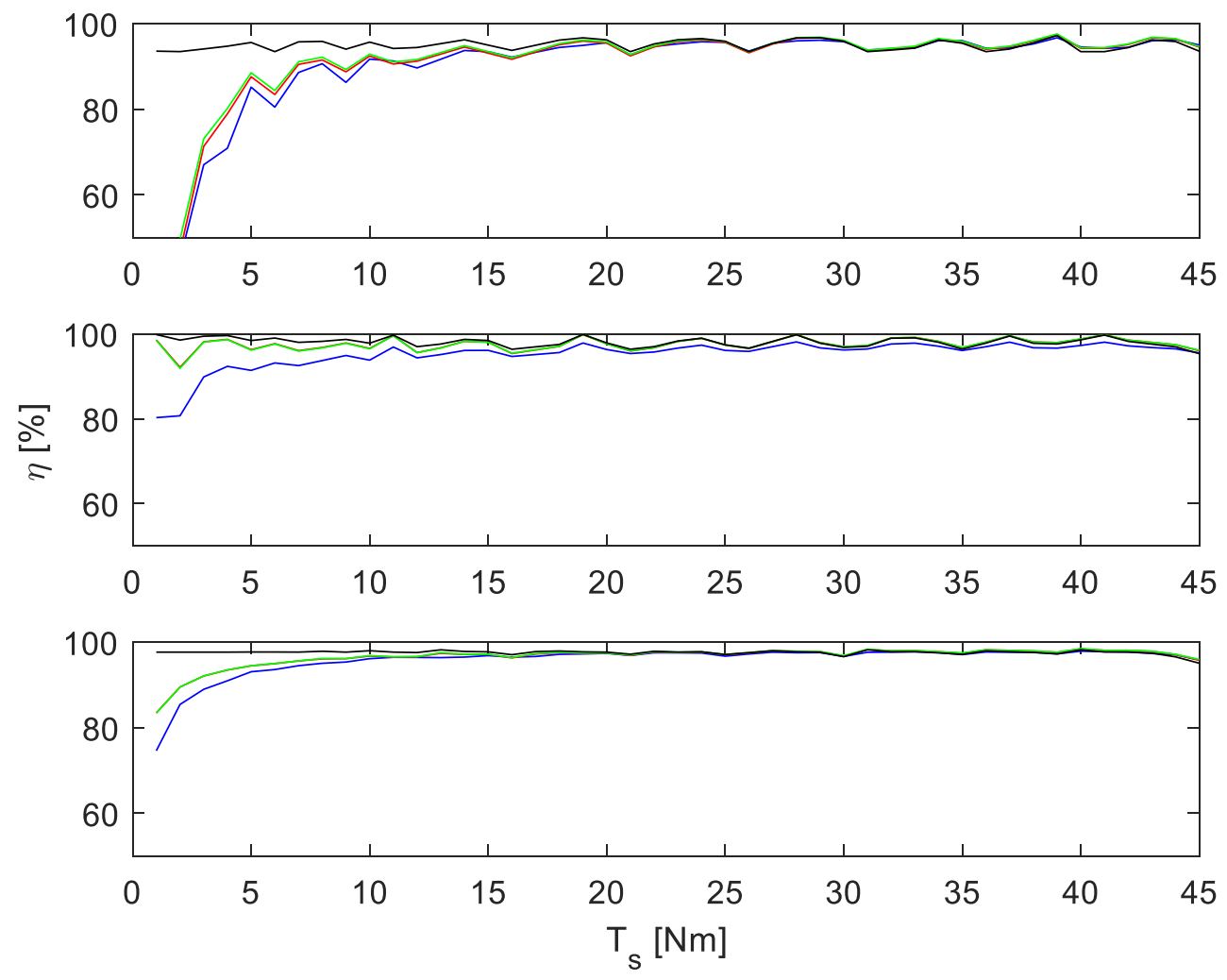

Figure 8: Efficiency of the planetary gear for varying torque applied to the sun. (a) For a speed ratio of 0 . (b) For a speed ratio of 1 . (c) For a speed ratio of 2 . Blue: measurement, red: case 1, green: case 2, black: case 3 .

\section{Conclusion}

In this paper a method is described to estimate the efficiency of a 2-DOF planetary gearbox over a wide range of operating points. The model combines the theory of virtual power with a limited set of measurements. These measurements are conducted to calculate the efficiency of 2 power paths: between sun \& carrier and carrier \& ring. It is shown that these efficiency terms merely depend on the load torque. The required measurement time is thus limited as the variation with speed does not have to be taken into account. The results show the good match between measured efficiency of the planetary gearbox and the estimated value. Average difference between both is $1.7 \%$ if the complete operating range is considered. This drops to less than $1 \%$ is torque values below $10 \mathrm{Nm}$ are excluded.

\section{Acknowledgement}

This work was carried out for the EMTechno project (project ID: IWT150513) supported by VLAIO and Flanders Make, the strategic research center for the manufacturing industry. 


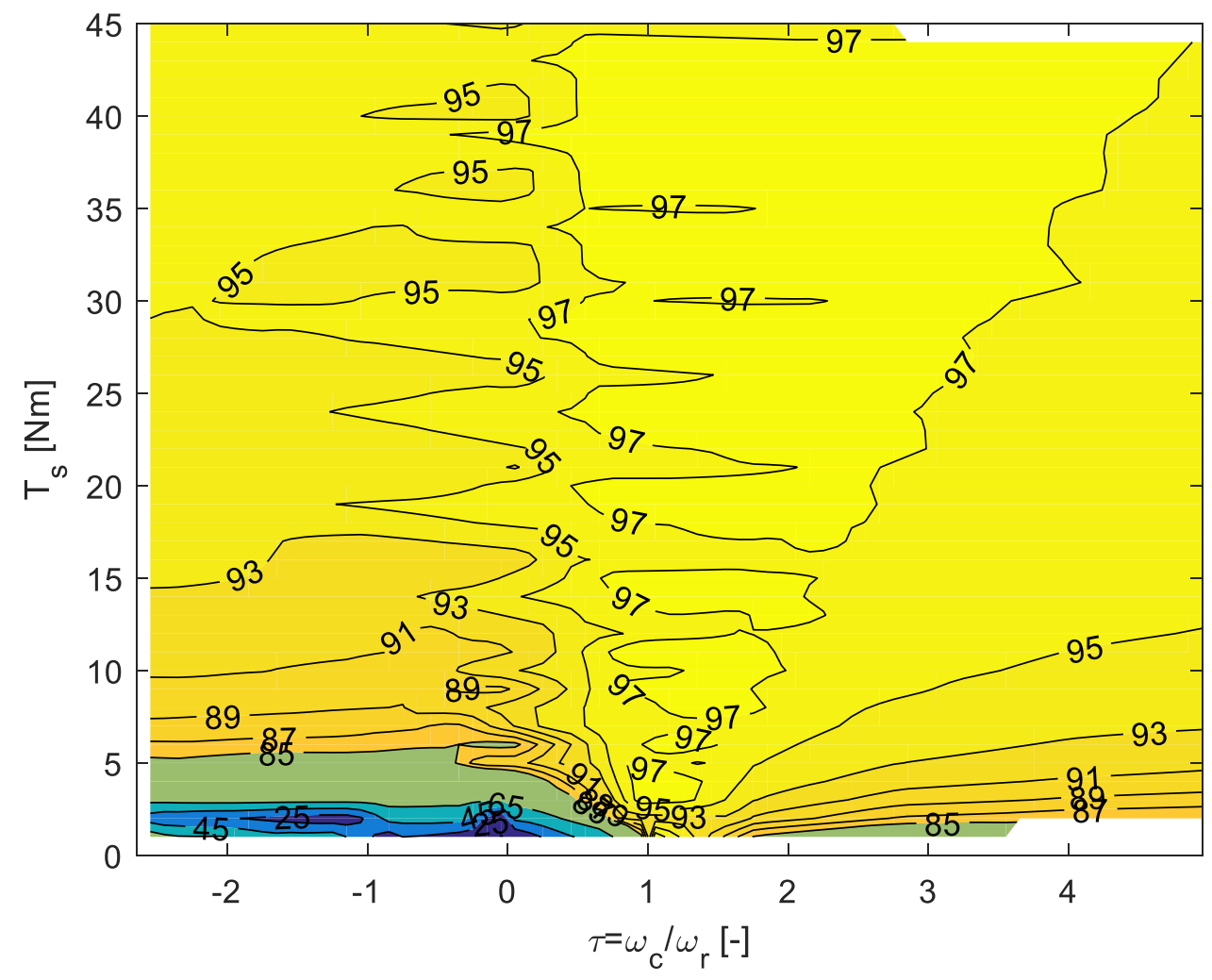

Figure 9: Simulated efficiency of the planetary gearset for varying speed ratio $\tau=\frac{\omega_{c}}{\omega_{r}}$ and stator torque $T_{s}$.

\section{References}

[1] S. Dereyne, P. Defreyne, E. Algoet, and K. Stockman, "Construction of an energy efficiency measuring test bench for belt drives Test bench setup," in Energy efficieny of Motor Driven Systems, 2013, pp. 1-8.

[2] S. Dereyne, P. Defreyne, E. Algoet, and S. Derammelaere, "Efficiency measurement campaign on gearboxes," in Energy efficieny of Motor Driven Systems, 2015, pp. 1-11.

[3] E. Vinot, R. Trigui, Y. Cheng, C. Espanet, A. Bouscayrol, and V. Reinbold, "Improvement of an EVT-Based HEV Using Dynamic Programming," IEEE Trans. Veh. Technol., vol. 63, no. 1, pp. 40-50, 2014.

[4] C. Nutakor, A. Klodowski, J. Sopanen, A. Mikkola, and J. I. Pedrero, "Planetary gear sets power loss modeling: Application to wind turbines," Tribol. Int., vol. 105, no. May 2016, pp. 42-54, 2017.

[5] J. D. de Gevigney, F. Ville, C. Changenet, and P. Velex, "Tooth friction losses in internal gears: Analytical formulation and applications to planetary gears," Proc. Inst. Mech. Eng. Part J J. Eng. Tribol., vol. 227, no. 5, pp. 476-485, 2013.

[6] E. Pennestri and P. P. Valentini, "A review of formulas for the mechanical efficiency analysis of two degrees-of-freedom epicyclic gear trains," J. Mech. Des., vol. 125, no. 3, pp. 602-608, 2003.

[7] C. Chen, "Power flow and efficiency analysis of epicyclic gear transmission with split power," Mech. Mach. Theory, vol. 59, pp. 96-106, 2013. 
[8] K. Davies, C. Chen, and B. K. Chen, "Complete Efficiency Analysis of Epicyclic Gear Train With Two Degrees of Freedom," J. Mech. Des., vol. 134, no. 7, p. 071006, 2012.

[9] C. Chen and J. Angeles, "Virtual-Power Flow and Mechanical Gear-Mesh Power Losses of Epicyclic Gear Trains," J. Mech. Des., vol. 129, no. January 2007, pp. 107-113, 2007.

[10] C. Chen and J. Chen, "Efficiency analysis of two degrees of freedom epicyclic gear transmission and experimental validation," Mech. Mach. Theory, vol. 87, pp. 115-130, 2015.

[11] S. Dereyne, E. Algoet, P. Defreyne, and K. Stockman, "An energy efficiency measurement test bench for gearboxes," in Energy efficieny of Motor Driven Systems, 2013. 